\title{
Social Choice Theory and the Informational Basis Approach ${ }^{*}$
}

\author{
Kevin Roberts \\ Nuffield College \\ Oxford \\ kevin.roberts@nuf.ox.ac.uk
}

This version: March 2006

\begin{abstract}
:
For over a quarter of a century, the use of utility information based upon interpersonal comparisons has been seen as an escape route from the Arrow Impossibility Theorem. This paper critically examines this informational basis approach to social choice. Even with comparability of differences and levels, feasible social choice rules must be insensitive to a range of distributional issues. Also, the Pareto principle is not solely to blame for the inability to adopt rules combining utility and non-utility information: if the Pareto principle is not invoked then there is no way of combining utility and non-utility information in a ranking of states unless levels of utility are comparable; with only level comparability, information must be combined in restrictive ways and the notion of giving different independent weight to different considerations is ruled out. If informational bases are viewed as the restriction on information that is available, rather than a theoretical limit on information, then there exist methods to estimate richer informational structures and overcome some of these difficulties.
\end{abstract}

*The author is grateful to Marc Fleurbaey for his comments. 


\section{Introduction}

Social choice theory is concerned with the principles underlying choice and preference when a group of individuals have different preferences over the options available. In the sense that it is thought desirable to reflect individual preference in a group preference, social choice theory deals with the principles of aggregation of preference. At this general level, social choice theory is applicable to decisions making by committees, the political voting process, and most aspects of welfare economics.

In narrower terms, social choice theory is concerned with the formal analysis of the aggregation of information to generate a social choice or preference. The basic building block for this theory is the work of Kenneth Arrow (1951), the generalization to permit an understanding of how the aggregation of information is sensitive to the nature of information available is due to Amartya Sen, most notably in his book Collective Choice and Social Welfare (Sen (1970)). This book remains a tour de force in terms of being both a map to guide researchers and an inspiration to them in their endeavours. Indeed, the flowering of the subject in the 1970s and later is due directly to clarity of the issues that Sen laid out in that book.

The purpose of this essay is to critically examine the informational basis approach to social choice as developed by Sen. ${ }^{1}$ The major finding of Arrow - his famous General (Im)possibility Theorem - is that aggregation in the social choice is impossible if the aggregation process is to satisfy a set of 'reasonable' conditions. Sen's work points to the informational restrictions inherent in the Arrow set-up and shows that aggregation with richer information permits social choices to be made with more 'appealing' mechanisms. In particular, this richer information may include utility or individual welfare information that is interpersonally comparable. The nature and extent of this comparability is a major focus of the subject and of this essay.

\footnotetext{
${ }^{1}$ In the field of social choice, broadly conceived, Sen has made many contributions across a wide range of fields. Within the field more narrowly conceived, one can separate out at least three sub-fields where his contribution has been fundamental - the conditions under which majority rule gives rise to an ordering of social states (Sen (1966), see also Sen and Pattanaik (1969)), the conflict between rights and Paretianism (Sen 1970a), and the generalization of the Arrow paradigm to social welfare functionals which is the subject of this essay.
} 
I start with a presentation of Arrows's approach to the analysis of social choice, based upon the use of a social welfare function, and of Sen's extension to this, based upon the use of a social welfare functional. I then consider alternative informational bases for interpersonal comparisons and the use of invariance transforms as pioneered in the social choice context by Sen. ${ }^{2}$

Given the nature of the original Arrow result, it is not surprising that Sen's approach has been viewed as a solution to its negativeness. However, part of the purpose of this essay is to demonstrate that conventional notions of comparability of utilities imply, through their informational parsimony, a restrictiveness in terms of mechanisms for social choice. This is particularly the case if comparability information is restricted because more detailed information is considered meaningless, rather than being considered meaningful but not available. Within a welfarist context, feasible mechanisms to generate social welfare from the aggregation of individual welfares remain restrictive. In a more general non-welfarist context, it will be shown that mild restrictions on utility information become very important when welfare information must be compared with non-welfare information. ${ }^{3}$ This will be demonstrated by exposing the difficulties of moving beyond welfarism, even when welfarist conditions like the Pareto criterion are not invoked.

\section{Social Choice Theory}

Social choice has a long history, dating back at least to ancient Greece. A recurring theme has been the difficulty of aggregating individual preferences into a social preference. This has been demonstrated by considering examples of situations where difficulties occur, the most well-known being the paradox of majority voting associated with Condorcet (1785): consider three individuals, individual 1 prefers social state or outcome $x$ to $y$ to $z$, 2 prefers $y$ to $z$ to $x$ and 3 prefers $z$ to $x$ to $y$ : a majority (1 and 3) prefer $x$ to $y$, a majority ( 1 and 2) prefer $y$ to $z$ and a majority (2 and 3) prefer $z$ and $x$. Thus, for any state, there is another

\footnotetext{
${ }^{2}$ Following Sen, the term informational basis is assumed to relate to the structure of individual utility or welfare information. A wider interpretation can also be adopted (Fleurbaey (2003)).

${ }^{3}$ It is not the purpose of this essay to examine Sen's work on the role of non-utility information and the conflict of rights and the Pareto principles (Sen (1970a)). Here, the conflict is less stark but it does not require the sledge-hammer of the Pareto principle (Sen (1979)) to expose the difficulties of adopting a pluralist approach, encompassing welfarist and non-welfarist principles.
} 
state preferred by a majority, and a ranking of states based upon majority rule is intransitive. Other voting methods would give more satisfactory results in this situation but possess other problems, again being demonstrated by invoking examples. The problem with analyses of this sort is that they do not move beyond a collection of examples and it is to Arrow (1951) that we owe the debt for creating a more general approach to the subject. In essence, he sought to move away from an investigation of how particular aggregation mechanisms worked in particular cases to how classes of mechanisms worked in all 'feasible' scenarios.

We lay out some formalities. Assume that we wish to construct a social ordering of social states. An ordering is complete (two states can be compared), reflexive (a state is as good as itself), and transitive (if $x$ is at least as good as $y$ and $y$ is at least as good as $z$ then $x$ is at least as good as $z$ ). The set of social states is $X$, and $x R y$, where $x, y \in X$, denotes the fact that $x$ is socially weakly preferred to $y$ ( $x I y$ denotes indifference and $x P y$ denotes strict preference). Arrow considers the aggregation of individual preferences which, themselves, are orderings over $X$. Thus, assume that there is a set of individuals $N$ and, for $i \in N, R_{i}$ denotes $i$ 's ordering of the social states. A social welfare function (SWF) $f$ is an aggregation mechanism, which determines a social ordering as a function of individual orderings: $R=f\left(\left\langle R_{i}\right\rangle_{i \in N}\right)$. The most obvious examples of social welfare functions are voting mechanisms like majority rule. It is inherent in the Arrow structure that individual utility information consists of individual orderings - there is no intensity of preference or ranking of utility levels across individuals.

In a particular situation or example, the individual preferences are specified and $f$ determines some social ordering. Arrow lays down four conditions that should be satisfied by a SWF:

(U) Unrestricted Domain: $f$ is defined for all possible individual orderings

(I) Independence: If $\left\langle R_{i}\right\rangle_{i \in N}$ and $\left\langle R_{i}^{\prime}\right\rangle_{i \in N}$ coincide over some pair of states $x$ and $y$ then $R=f\left(\left\langle R_{i}\right\rangle_{i \in N}\right)$ is the same as $R^{\prime}=f\left(\left\langle R_{i}^{\prime}\right\rangle_{i \in N}\right)$ over the pair $\{x, y\}$. 
(P) Pareto: If $x P_{i} y$ for all $i \in N$ then $x P y$ (where $P$ is derived from $R_{i}=f\left(\left\langle R_{i}\right\rangle_{i \in N}\right.$ ) and $R_{i}$ is each individual's ordering giving rise to the strict preference over $\{x, y\}$ ).

(ND) Non-Dictatorship: For each individual $d$, there exists a set of preferences $\left\langle R_{i}\right\rangle$ and a pair $\{x, y\}$ such that $x P_{d} y$ and $y R x\left(R=f\left(\left\langle R_{i}\right\rangle_{i \in N}\right)\right)$.

Arrow's impossibility theorem shows that there exists no SWF satisfying these four conditions, e.g. majority voting fails $(U)$ because in some situations the ranking it creates is not an ordering.

Condition $(U)$ demands that an ordering can be created for all individual preferences - it forces a consideration of counter-factuals so that a social ordering needs to be created not only for individual orderings that actually occur but also for hypothetical possibilities. ${ }^{4}$ Condition (I) relies upon the use of counter-factuals to ensure that the social choice between a pair $\{x, y\}$ is independent of the individual rankings of any other pairs of states it is by intention consequentalist. Condition $(P)$ is a welfarist condition pointing both to a desire to respect individual preference and a willingness to be guided only by individual preference when there is unanimity. Condition $N D$ requires that social preference does not reflect one person's individual preference irrespective of the preferences of other individuals.

In the Arrow framework, what is the information on which the social ordering is based? Utility information enters directly through the individual orderings of states. Other information enters through the description of each state which, for instance, serves to differentiate state $x$ from $y$. This information can be rich in terms of a description of society in each state but condition $(U)$ can only be reasonable if states $x$ and $y$ are described sufficiently loosely for individuals to have any preference between $x$ and $y$. It could be possible to say that individual $i$ has an adequate well-being in state $x$ as a description of state $x$ but it would not then be possible to say that $i$ 's well-being in state $y$ is inadequate,

\footnotetext{
${ }^{4}$ One can avoid the use of hypothetical possibilities if the set of social states is sufficiently large and the set of individual orderings are sufficiently rich, this richness extending to triples of states which are viewed as similar apart from with regard to individual preference over the triples. See Parks (1976). For aggregation based upon social welfare functionals as developed below, see Roberts (1980b).
} 
given that it is possible that $y R_{i} x$ and $(U)$ demands that it is reasonable to construct a social ordering in this situation. Thus, if condition $(U)$ is to be reasonable, non-utility information can enter into the primitive description of states in limited ways.

\section{Sen's Social Welfare Functional}

It is now a commonplace to view the Arrow problem as an attempt to determine a reasonable social ordering with too little, particularly utility, information. We owe this interpretation to Sen (1970). He proposes a generalization of the Arrow approach to make possible the incorporation of richer utility information. Utility information is captured by a numerical representation of utility so that $u(x, i)$ denotes utility in state $x$ of individual $i$. Instead of a SWF, we have a social welfare functional (SWFL) $f$ mapping utility representations into a social ordering: $R=f\left(\langle u(x, i)\rangle_{x \in X, i \in N}\right)$. The Arrow conditions can be straightforwardly amended to apply to SWFLs and condition (I), for instance, can be reformulated as

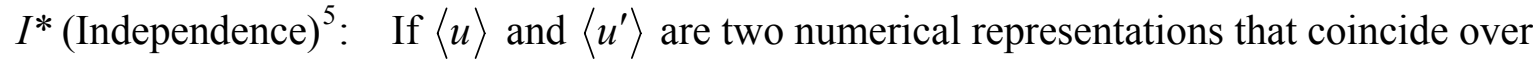
a pair of states $\{x, y\}$, i.e. $u(x, i)=u^{\prime}(x, i)$ and $u(y, i)=u^{\prime}(y, i)$ for all $i \in N$, then $R=f(\langle u\rangle)$ and $R^{\prime}=f\left(\left\langle u^{\prime}\right\rangle\right)$ coincide over $\{x, y\}$.

Conditions $(U),(P),(N D)$ can be similarly reformulated as $\left(U^{*}\right),\left(P^{*}\right)$ and $\left(N D^{*}\right)$.

The usefulness of using SWFLs is that utility information can incorporate more information than is contained in a simple individual ordering. The information content of utility information can be fine-tuned by imposing conditions which ensure that a SWFL treats different numerical representations as equivalent. Let $\mathcal{U}$ be the set of all real valued functions defined over $X x N$. Consider partitioning $\mathcal{U}$ into a collection of subsets $P_{1}, \ldots, P_{K}$ where $\mathcal{U}=\cup_{k=1, K} P_{k}$ and $P_{k} \cap P_{\ell}=\phi$ for all $k, \ell$. We call $P=\left\{P_{1}, \ldots \ldots, P_{K}\right\}$ a partition and let $\mathcal{P}$ be the set of all partitions of $u$. A partition captures the informational context of utilities and this suggests the definition

\footnotetext{
${ }^{5}$ We use an asterisk to denote that the condition applies to SWFLs.
} 
P-Invariance $\left(P-I^{*}\right): \quad$ A SWFL $f$ is P-invariant with respect to partitioning $P=\left\{P_{1}, \ldots \ldots, P_{k}\right\}$ if for all $u, u^{\prime} \in P_{k}$, for some $k, f(\langle u\rangle)=f\left(\left\langle u^{\prime}\right\rangle\right)$.

If the partitioning of $u$, call it $\tilde{P}$, is such that $u$ and $u^{\prime}$ are in the same partition if they induce the same ordering of states for each individual, i.e. for all $i \in N, x, y \in X: u(x, i) \geq u(y, i) \Leftrightarrow u^{\prime}(x, i) \geq u^{\prime}(y, i)$, then a SWFL that is P-Invariant with respect to $\tilde{P}$ ignores all utility information other than individual orderings. By Arrow's theorem, there is no such SWFL satisfying $\left(U^{*}\right),\left(I^{*}\right),\left(P^{*}\right)$ and $\left(N D^{*}\right)$ which is P-Invariant with respect to $\tilde{P}$.

The idea of capturing utility information as a partition of $\mathcal{U}$ is that utility functions in the same partition are informationally equivalent. The coarser the partition, the more demanding is P-Invariance.

\section{Information Bases of Interpersonal Comparability and Invariance Transforms}

In this section, we show how different types of interpersonal comparability can be captured using partitions generated through the use of invariance transforms. We go on to examine the extent to which richer informational structures as captured by finer partitions overcome the pessimism generated by the Arrow Impossibility Theorem.

In terms of utility information, it is usual to view utilities as being ordinal or cardinal.

Looking first at just one individual's utility function, ordinality captures the idea that utility information defines an ordering of states - the levels of utility can be ordered - but no more. Thus, the primitive notion is an individual ordering. This ordering can be given a numerical utility representation by assigning higher utility to more preferred outcomes and the arbitrariness of the particular representation is captured by P-Invariance with the 
partitioning $\tilde{P} .^{6}$ An alternative method of defining equivalence of utility functions is to use the idea of an invariance transform $<\phi_{i}>$ which has the property that $\phi_{i}(u(\cdot, i))$ is equivalent to $u(\cdot, i)$ - under ordinalism, $\phi_{i}$ can be any strictly monotonic transformation. ${ }^{7}$

Ordinality allows levels of utility to be compared. Cardinality allows utility differences to be compared: the ordering of $u(x, i)-u(y, i)$ over the set $X x X$ is the same for all utility functions with the same information partition. In terms of an invariance transform, $\phi_{i}$ is an affine strictly increasing affine transformation: $\phi_{i}\left(u_{i}\right)=\alpha_{i}+\beta_{i} u_{i}$ where $\beta_{i}>0$.

The informational content of utility functions becomes interesting when there is comparability across individuals (Sen $(1970,1977)$ ). Levels of utility are comparable, e.g. the statement that individual $i$ in state $x$ is better off than $j$ in state $y$, if the partitioning of the utility space distinguishes between two utility functions $u(\cdot, \cdot)$ and $u^{\prime}(\cdot, \cdot)$ with a different ordering in $X x N$ space. Thus level comparability is an ordinal notion and an example of a SWFL satisfying $\left(U^{*}\right),\left(I^{*}\right),\left(P^{*}\right)\left(N D^{*}\right)$ and $(P-I)$ with this information partition is a Rawlsian maximin rule which ranks states according to the well-being of the worst-off in each state.

If differences in utility are comparable then $u(x, i)-u(y, i)$ can be ordered in $X x X x N$ space, e.g. statements of the form that $i$ gains more from the move from $x$ to $y$ than $j$ gains from the move from $w$ to $z$ can be made. This is a cardinal notion and, in terms of invariance transforms, it is necessary that if $\phi_{i}\left(u_{i}\right)=\alpha_{i}+\beta_{i} u_{i}$ and $\phi_{j}\left(u_{j}\right)=\alpha_{j}+\beta_{j} u_{j}$ then $\beta_{i}=\beta_{j}$. Comparability of differences - often termed unit comparability - underlies what is necessary for the implementation of a utilitarian rule.

Comparability of levels and differences is not, of course, an either/or. If there is both an ordering of levels and a compatible ordering of utility differences then we have, what Sen

\footnotetext{
${ }^{6}$ If the number of states is a continuum then some continuity assumption is required to ensure the existence of a numerical representation. With cardinal utility representations, there are extra technical demands (Basu (1983), Roberts (1997)).

${ }^{7}$ For a set of invariance transforms $\Phi_{i}$ to define a partitioning of the utility space, it is required that i)

$\Phi_{i}$ contains the identity transform; ii) if $\phi_{i} \in \Phi_{i}$ then $\phi_{i}^{-1} \in \Phi$ and iii) $\phi_{i}^{\prime} \in \Phi_{i}$ and

$\phi_{i}^{\prime \prime} \in \Phi_{i} \Rightarrow \phi_{i}^{\prime}\left(\phi_{i}^{\prime \prime}\right) \in \Phi_{i}$.
} 
$(1970,1970 b)$ has termed, full comparability. If this is to be captured by the use of invariance transforms then $\phi_{i}$ must take the form $\phi_{i}\left(u_{i}\right)=\alpha+\beta u_{i}, \beta>0$, where $\alpha$ and $\beta$ are independent of $i$.

We can add more comparability above and beyond full comparability. For instance, it may be possible to compare the level of individual utility with some independent norm which may allow one to make judgements about the value of increasing the population size, e.g. a population increase may be desirable if the 'new' individuals have a utility above the norm. This is termed ratio-scale comparability. If there is an independent norm, the utility representation can be chosen to ensure that the norm has zero utility and, in terms of invariance transforms, we will have $\phi_{i}\left(u_{i}\right)=\beta u_{i}$ where $\beta>0$ is independent of $i$. In the limit, we could move to a degree of comparability that implied that each partition consisted of one element. To obtain this from an ordering it would be necessary to rank outcomes against a set of independent norms or, more concretely, an independent 'yardstick'. Let us call this total comparability.

\section{Aggregation Possibilities}

A generally accepted view is that whilst there is no acceptable method of aggregation based upon the use of individual non-comparable orderings, a wide range of possibilities is opened up when richer informational structures are permitted.

Assume that a reasonable aggregation rule satisfies $\left(U^{*}\right),\left(I^{*}\right),\left(P^{*}\right)$ and $\left(N D^{*}\right)$. If utility functions are cardinal but not comparable then utility differences are intrapersonally comparable. However, this information relates to the utility that an individual achieves in at least three states, e.g. the difference in utility between $x$ and $y$ and between $x$ and $z$, say. By $\left(I^{*}\right)$, this is ruled out as admissible information that can influence the aggregation rule. Thus, cardinality without comparability still gives rise to impossibility, a first (negative) characterization result, proved by Sen (1970), which moved beyond the Arrow framework.

To make progress, we need to use interpersonally comparable information. If there is comparability of differences, but no more, then the set of aggregation rules satisfying ( $\left.U^{*}\right)$, $\left(I^{*}\right),\left(P^{*}\right)$, and $\left(N D^{*}\right)$ is the set of weighted utilitarian functions with different individual 
utility functions being given different weights. This important characterization result is proved by d'Aspremont and Gevers (1977). If there is a desire to treat individuals symmetrically - a condition of anonymity - then the only admissible aggregation rule is the (unweighted) utilitarian rule. These rules apply when the weighted sum of utilities is strictly greater in one state than another; when the sum is the same in the two states, it is possible that the ranking can depend upon non-utility information serving to define social states (Roberts (1980a)).

If there is comparability of levels, but no more, then the set of aggregation rules take the form of associating the welfare of any state with the utility level of one individual in that state, the individual in each state being determined by who is the person and/or the position of the individual in the hierarchy of utilities in that state (Roberts (1980)). If the symmetry condition of anonymity is added then welfare is associated with the utility level at some position in the utility hierarchy (Gevers (1979)). Adding a minimal equity condition, which says that individuals with lower utility should be given at least as much weight as those with higher utility, ensures that the position in the hierarchy on which to focus is the individual with lowest utility (Hammond (1975), Strasnick (1976)). We are thus led to the conclusion that interpersonal comparability of levels, but no more than this, leads to a Rawlsian maximin aggregation rule.

Assume that there is both comparability of differences and comparability of levels so we have full comparability, an informational structure that economists, at least, seem to consider rich. With such comparability, there is no information which tells us that we are discussing a society where everybody is subject to abject poverty or to unlimited riches adding a constant to everybody's utility does not change comparisons of levels or of differences. Any aggregation mechanism must be insensitive to this concern. Relatedly, the aggregation mechanism must be insensitive to the extent of utility inequality as utility differences can be scaled upwards or downwards. In particular, aggregation mechanisms cannot be more sensitive to inequality, the greater it becomes. For instance, if, in a twoperson society, it is determined that the utility vector $(10,10)$ gives as much social welfare as $(8,14)$ then, by admissible scaling of utilities, these vectors give also the same social welfare as $(9,12)$, using a scaling factor $\beta=1 / 2$, or $(6,18)$, using a scaling factor $\beta=2 .^{8}$ In

\footnotetext{
${ }^{8}$ The class of possible aggregation mechanisms under full comparability is characterized in Roberts (1980a). If one also imposes a separability condition which states that the social ordering over a pair of social states $x$ and $y$ should not be sensitive to utility information of individuals whose well-being is the
} 
particular, it can be seen that attitudes to small deviations from equality are fully inherited by the attitudes to large deviations from equality. ${ }^{9}$ These considerations are unimportant to a utilitarian or to a welfarist Rawlsian, the utilitarian being unconcerned with utility inequality, the Rawlsian showing maximum concern at all times. But to implement a rich class of aggregation mechanisms, with attitudes to inequality depending upon the extent of inequality, the informational requirements seem to demand the existence of an independent yardstick. Thus, the use of interpersonal comparisons may allow one to escape from the straitjacket of the Arrow result but the informational basis approach to social choice points to the restrictiveness of possible aggregation mechanisms, restrictiveness coming from the 'technical' limitations of the information on which judgments can be based.

\section{Utility Information in Interpersonal Comparisons}

The social welfare functional (SWFL) approach is based upon individual well-being information being captured by a utility function. To economists, the usual use of a utility function is as a way of representing a preference ordering with any meaning to the notion of utility coming entirely from the nature of the preference order being represented. With an ordinal ordering, the utility function is a labelling of states; with cardinal orderings there are restrictions on the orderings which permit representation (Basu (1983), Roberts (1997)) and these restrictions are reasonable given that the cardinal ranking relates to a ranking of differences.

We have seen in section 4 that utility information in its primitive form can be captured by an ordering of utility levels and/or utility differences. It may therefore appear more natural to consider SWFLs as a function of these orderings directly rather than through the intermediate step of creating a representative utility function. Here we show that the use of this intermediate step has some hidden implications.

\footnotetext{
same in the two states, then the only possible aggregation mechanisms involve a focus on the worst-off, as in a maximin, a focus on the best-off, or a utilitarian focus on total utility. The details of this characterization are in Deschamps and Gevers (1978).

${ }^{9}$ This implies that iso-welfare curves in utility space must be cones emanating from points of full equality. See Roberts (1980a).
} 
Consider an example where interpersonal comparability takes the form of difference comparability and, over a pair of states, $x$ and $y$, a three individual society exhibits the following difference orderings

$$
u(x, 1)-u(y, 1)>u(y, 2)-u(x, 2)>u(y, 3)-u(x, 3)>0
$$

Here, the placement of zero is information derivable from the preference ordering of states of each individual. Would a utilitarian prefer $x$ to $y$ or vice versa? Individual 1 gains more in the move from $y$ to $x$ than individuals 2 and 3 each lose but the 'cumulative' loss of 2 and 3 may dominate 1's gain. Thus if we use a SWFL based upon comparability orderings and we invoke an independence condition, call it $\left(I^{* *}\right)$, that makes the social ranking over $\{x, y\}$ independent of difference orderings involving states other than $\{x, y\}$ then, in this example, a utilitarian rule would not be implementable. ${ }^{10}$ So, if an unrestricted domain condition $\left(U^{* *}\right)$ is imposed requiring a SWFL to create a social ordering in all cases then the utilitarian rule is not an admissible SWFL. In fact, we can prove an interesting result for a world where the informational basis is a ranking of utility differences (where $\left(P^{* *}\right)$ and $\left(N D^{* *}\right)$ are the marginally reformulated versions of $\left(P^{*}\right)$ and $\left.\left(N D^{*}\right)\right)$ :

Theorem The only SWFL that satisfies $\left(\mathrm{U}^{* *}\right),\left(\mathrm{I}^{* *}\right),\left(\mathrm{P}^{* *}\right)$ and $\left(\mathrm{ND}^{* *}\right)$ is a weighted utilitarian rule giving equal weight to two individuals $\left(\mathrm{d}_{1}\right.$, and $\mathrm{d}_{2}$, say) and zero weight to all other individuals.

Proof Any rule satisfying $\left(\mathrm{U}^{* *}\right),\left(\mathrm{I}^{* *}\right),\left(\mathrm{P}^{* *}\right)$ and $\left(\mathrm{ND}^{* *}\right)$ is a rule satisfying $\left(\mathrm{U}^{*}\right),\left(\mathrm{I}^{*}\right),\left(\mathrm{P}^{*}\right)$ and $\left(\mathrm{ND}^{*}\right)$ under the invariance transform of difference comparability. From d'Aspremont and Gevers (1977), any such rule must take the form of ranking states according to a weighted sum of utilities. Assume that at least three individuals are given positive weight. Label these three individuals $1,2,3$, their labelling relating to their weight in the weighted sum of utilities: $\gamma_{1} \leq \gamma_{2} \leq \gamma_{3}$. Assume that $u(x, i)=u(y, i)$ for all $i \neq 1,2,3$ and let 1,2 and 3 's utility information over the pair $\{x, y\}$ be as in $(\dagger)$ above. What is the weighted sum of utilities over $\{x, y\}$ in this case? Compatible with $(\dagger)$ is $u(x, 1)-u(y, 1)$ being sufficiently positive (compared to the other utility differences) so that the weighted sum of utilities is higher in state $x$ than $y$. Thus $x P y$ in scenario ( $\dagger$. However, compatible with $(\dagger)$ is a case

\footnotetext{
${ }^{10}$ See Bossert (1991).
} 
where the three utility differences are equal so that, as $w_{2}+w_{3}>w_{1}$ the weighted sum of utilities is higher in state $y$. We have thus shown that the weighted utilitarian rule with three individuals being given weight cannot be implemented based upon an ordering of utility differences. Now assume that only two individuals, 1 and 2, are given weight and that $\left(\gamma_{2}>\gamma_{1}\right)$. When the ranking of differences as in $(\dagger)$, the weighted sum of utilities is higher under $y$ when $u(x, 1)-u(y, 1)$ is close to $u(y, 2)-u(x, 2$,$) because \gamma_{2}>\gamma_{1}$, and the weighted sum is higher under $x$ when $u(x, 1)-u(y, 1)$ is large compared to $u(y, 2)-u(x, 2)$. Again, the weighted utilitarian rule is not implementable. If only one individual has positive weight then $\left(\mathrm{ND}^{* *}\right)$ is directly violated so we are left with the possibility that two individuals, 1 and 2, have equal weight and all others have zero weight. If $\operatorname{sign}(u(x, 1)$ $u(y, 1))=\operatorname{sign}(u(x, 2)-u(y, 2))$ then the weighted sum of utilities is unambiguous. Otherwise, for some labelling of the two individuals and the two states, we have $u(x, 1)$ $-u(y, 1)>u(y, 2)-u(x, 2)>0$ and the weighted sum of utilities is always higher in state $x$ : thus weighted sum of utilities is implementable in this case and the proof is completed.

Whilst a oligarchy of two individuals awards a dictatorship it is not much of an improvement for a society of perhaps many million!

The theorem above shows the possibilities when the available information is a ranking of utility differences. However, the standard approach to aggregation with information based upon utility differences does not give this result. With a SWFL based upon utility information and this information incorporating difference comparability, i.e. with invariance transforms of the form $\phi_{i}\left(u_{i}\right)=\alpha_{i}+\beta u_{i}, \beta>0$, inducing the invariance partitions of the utility space, we know from d'Aspremont and Gevers (1977) that $\left(U^{*}\right),\left(I^{*}\right)$, $\left(P^{*}\right),\left(N D^{*}\right)$ and $\left(P-I^{*}\right)$ for this information structure defines the class of admissible SWFLs to be the class of weighted utilitarian functions: the social ordering is determined by the ordering of $\sum_{i \in N} \gamma_{i} u(\cdot, i)$, for appropriate weights $\gamma_{i} \cdot{ }^{11}$ How is the difference between the two results to be explained? The important source of this difference is that utility information is created from the ranking of utility differences and utility information relating to a pair of states $\{x, y\}$ can incorporate information about utility differences

\footnotetext{
${ }^{11}$ The Pareto condition implies that all the $\gamma_{i}$ are non-negative and at least two must be positive. The weights are otherwise unrestricted.
} 
involving states other than $x$ and $y$. To see this, consider again the three-person example given in $(\dagger)$. Assume that among all other states, there is a state $z$ such that

$$
u(z, 2)-u(y, 2)=u(y, 3)-u(x, 3)
$$

Knowing this, it is now possible to compare the sum of utilities over the pair $\{x, y\}$. We have:

$$
\begin{aligned}
\sum_{i=1,3}[u(x, i)-u(y, i)] & =u(x, 1)-u(y, 1) \\
& +u(x, 2)-u(y, 2) \\
& -u(z, 2)+u(y, 2) \\
& =(u(x, 1)-u(y, 1)) \\
& -(u(z, 2)-u(x, 2)) .
\end{aligned}
$$

Thus, the ranking of the sum can be computed by information determined by the ordering of utility differences. But to determine information relating to utility differences over $\{x, y\}$ it is necessary to consider utility differences relating to another state $z$ - the ordering of differences over all pairs of states provides utility information relating to states $x$ and $y$. If the problem is sufficiently rich, in the sense that states like state $\mathrm{z}$ in this example always exist, then, subject to the invariance transform for utility differences, a utility function will be uniquely defined and a weighted utilitarian ranking can always be determined.

In the SWFL approach based upon utility functions, the independence condition is subtly different to an independence condition which only allows information relating to $\{x, y\}$ to determine the social ranking over $\{x, y\}$. Condition $\left(I^{*}\right)$ demands that only utility information over $\{x, y\}$ is used to determine the social ranking over $\{x, y\}$. However, the ranking of utility differences involving states other than $\{x, y\}$ is used to create the utility function over $\{x, y\}$ : rankings involving other alternatives is used to inform what can be inferred about utilities over $\{x, y\}$. Consequentalism in a strict sense, as captured by $\left(I^{* *}\right)$ for instance, is foregone but the motivation for looking at alternative states is fully compatible with the thrust of consequentalism. It is not widely understood that this approach relies upon a degree of non-independence which permits information relating to 'irrelevant' alternatives to be relevant. 


\section{Meaningful Comparisons and the Information for Comparisons}

Robbins (1935) famously asserted the meaninglessness of interpersonal comparisons and the influence of this idea still casts a shadow over welfare economics. Excluding meaningless in a technical sense, which could relate to comparisons that failed a rationality test like transitivity, there still remains a notion of meaningless which relates to how we comprehend statements of comparison. For instance, it may not be difficult to comprehend an ordinal comparison of the form that $A$ is better off than $B$ whereas to say that $A$ is three times as well off as $B$ lacks such comprehension. Part of the problem here relates to the introduction of numerical quantities to compare aspects of well-being. But if, say, an ordering based upon ratio-scale comparisons is accepted then, by using such comparisons over the set of all social states and individuals, this would, through a similar path to that followed in the last section, allow numerical comparisons of relative well-being to be created. However, numerical comparisons, whilst being an implication of ratio-scale comparisons, could, because they are not part of normal vocabulary, be avoided in the presentation of individual utility information. It is a presentational matter only: if ratioscale comparisons can be made then aggregation rules based upon (relative) numerical comparisons of utility can be implemented.

However, despite this, the basic general point remains: it is easier to accept that somebody could find ratio-scale comparisons incomprehensible than that they would find interpersonal comparisons of utility differences incomprehensible.

\subsection{Informational Bases as Available Information}

Another interpretation of alternative information bases is that they relate to the information that is available to be aggregated. Thus, there may be a desire to implement a utilitarian rule, and this requires comparability of differences, but the information available takes the form of non-interpersonally comparable individual preference orderings. There are at least two approaches that can be taken. First, there is the conservative approach which gives up on the idea of generating a complete social ordering and, instead, only ranks two states if, with the information available, it is clear that the sum of utilities is at least as high in one 
state than in the other. In the present example, this would mean that states would be ranked only if they were comparable under the Pareto criterion.

The alternative, more radical, approach is to use available information to estimate a ranking of utility differences. In the present example, one approach is to take each individual's preference ordering and assign a utility of zero to the worst state, a utility of unity to the next worst, two to the next worst, and so on. If a utilitarian rule is applied to this information then the aggregation procedure is the well-known Borda rule of voting. Here, note that condition $(I)$ is violated because information from other states is used to estimate utility information for each pair of states. However, once this is done, the aggregation rule that is utilized is avowedly consequentalist. We note that there are many methods of using preference orderings to estimate difference comparable information. This contrasts with what was uncovered in the last section where information from other states enriches the information related to a pair of states but it does so non-conjecturally.

The Borda rule is one way of creating interpersonally comparable welfare differences. Without further information beyond preference orderings, the utility difference between two adjacently ranked states is as likely as not to be greater than the utility difference between any two other adjacently ranked states. The expected utility difference in the two circumstances is the same and so the Borda rule may be viewed as the expression of the judgement of a utilitarian expected welfare maximizer faced with very limited information. Similarly, if level comparable information is available then a utility of zero can be assigned to the lowest utility level faced by any individual in any state, unity to the second lowest level faced by any individual in any state, and so on (Sen (1977)). Here, the utility difference between two adjacently ranked states for some individual will vary with comparability across individuals being used to estimate the cardinal ranking for the single individual.

As well as using utility information in other states to estimate utility information relating to a pair of states, there is also the possibility of using non-utility information incorporated into the description of social states. For instance, individual income is often used as an indicator of well-being. If the description of states includes individual income information then this may be combined with available utility information to enhance the quality of utility information. However, the reasonableness of this approach is in conflict with the 
unrestricted domain condition $\left(U^{*}\right)$ which requires that the SWFL determines a social ordering for all utility information. If the description of social states provides information that is useful to enhance the quality of utility information then it is doubtful, for instance, that all individual orderings of the states can be feasible. ${ }^{12}$ Formally, condition $\left(U^{*}\right)$ becomes a condition that one would not wish to impose on aggregation mechanisms.

Consider an example where social states include a description of the observable status of individuals, including social variables, e.g. health status, and economic variables, like income. Let us accept a restricted domain condition which takes the form that, holding other variables constant, an individual is better-off in states where his income is higher. Assume that available information is ordinal and non-comparable, captured for individual $i$ by a utility function $u\left(y_{i}, s_{i}, i\right)$ where $y_{i}$ is $i$ 's income and $s_{i}$ is a vector of observable nonincome variables. The utility function serves to provide information about the trade-off in well-being for $i$ between income and other variables.

If the domain of the utility function is sufficiently unrestricted then it is possible to discover the income level which, in some benchmark situation $s^{*}$, gives $i$ the same utility level as in any particular social state. Define $m\left(y_{i}, s_{i}, i\right)$ as follows:

$$
u\left(y_{i}, s_{i}, i\right)=u\left(m\left(y_{i}, s_{i}, i\right), s^{*}, i\right)
$$

The function $\mathrm{m}$ is an ordinal representation of $i$ 's utility - a money metric utility function. If $s *$ is fixed then any welfare function as a function of these money-metric utilities can be implemented. For instance, in a two-person world, consider the welfare function (where $m_{i}$ is money-metric utility of individual $i$ )

$$
W=m_{1}+m_{2}+m_{1}^{2}+m_{2}^{2}
$$

This welfare function would not be implementable if, for instance, the utility functions were provided by utility information based upon ratio-scale comparisons - the ranking between $\left(b m_{1}, b m_{2}\right)$ and $\left(b \tilde{m_{1}}, b \tilde{m_{2}}\right)$ is not independent of the scale factor $b$. Let $m_{1}=1$, $m_{2}=5, \tilde{m_{1}}=3, \tilde{m_{2}}=4$. Then both $\left(m_{1}, m_{2}\right)$ and $\left(\tilde{m_{1}}, \tilde{m_{2}}\right)$ give a welfare of 32 . However, if the

\footnotetext{
${ }^{12}$ If non-utility information is used to update beliefs then, formally, it is possible that utility and nonutility information are statistically dependent even though the supports of the distribution functions underlying beliefs are independent.
} 
utilities are scaled by $b=3 / 2,\left(b m_{1}, b m_{2}\right)$ gives higher welfare, if they are scaled by $b=3$, $\left(b \tilde{m_{1}}, b \tilde{m_{2}}\right)$ gives higher welfare.

The example demonstrates that non-utility information and, in particular, information from other social states, can be used to "create" utility information with none of the measurability and comparability restrictions as discussed in Section 4 above. ${ }^{13}$ The welfare function described above may be rejected because it is based upon meaningless comparisons but, if such comparisons are considered to be meaningful then non-utility information can be used to estimate the information that is necessary to implement such a function. The money-metric utility function is an example of a created utility function embodying measurability and comparability characteristics different from the original utility information. Again, as with the utilitarian justification for the Borda rule, the created utility function involves a degree of conjecture; in the present example, this relates to a relationship between income and utility.

\subsection{Partial Comparability}

Once it is accepted that utility information is incomplete, there exists the possibility that available information may be more informative than is implied by being complete with respect to one information base, and incomplete with respect to some richer base. For instance, some but not all utility differences may be interpersonally comparable with individual preference orderings being complete. As Sen has put it, 'We may, for example, have no great difficulty in accepting that Emperor Nero's utility gain from the burning of Rome was smaller than the sum-total of the utility loss of all the other Romans who suffered from the fire' (Sen (1999)).

The analysis of this situation has been initiated by Sen $(1970,1970 \mathrm{~b})$ who uses a utility function approach and focuses on the case where comparability of differences would constitute complete information. Thus, if $u(x, i)$ is a utility function representation of available information and

\footnotetext{
${ }^{13}$ The use of data about income or expenditure and social variables is one route to take when faced with a recognized lack of welfare information (Sen (1999)). The example uses information from other states which give the same utility level to individuals as the state under consideration (see Pazner (1979)).
} 


$$
u^{\prime}(x, i)=\alpha_{i}+\beta u(x, i)
$$

for all $x, i$ and for some $\alpha_{i}$ and $\beta>0, u$ and $u^{\prime}$ capture equivalent information. As comparability of differences constitutes complete information, wishing to treat all individuals symmetrically would imply that the preferred aggregation mechanism would be utilitarianism.

Sen capures incomplete information by specifying information as a subset $L$ of all utility functions defined over $X x N$. If $u(.,$.$) is a member of L$ then, as comparability of differences gives the richest information structure, $u^{\prime}(.,$.$) as defined above will be a member of L$. However, $L$ may extend beyond such functions. For instance, it may include all utility functions of the form

$$
u^{\prime \prime}(x, i)=\alpha_{i}+\beta_{i} u(x, i)
$$

where $b \leq \beta_{i} / \beta_{j}$, for all $i, j$ and some $b \leq 1$. If $b=1$ then the set does not extend beyond the set defined by the $u^{\prime}$ functions; if $b$ is close to zero then the set of $u^{\prime \prime}$ functions preserve only the cardinality of individuals' utility functions and interpersonal comparability is lost. The subset $L$ can be thought of as being created from a primitive ranking of utility differences which will rank only in some circumstances (recall the Emperor Nero example above). Using the (incomplete) ranking, the set $L$ will be determined as the set of all utility functions compatible with the ranking.

Unlike the case of complete information, where the set of all utility functions is partitioned into subsets where any two utility functions in the same partition capture equivalent information, each subset $L$ captures the full extent of incompleteness as well as features of the information base that would be implied by complete information. This means that two different sets $L$ and $L^{\prime}$ of information can intersect. For instance, an individual may prefer state $w$ to $x$ to $y$ to $\mathrm{z}$. Let $L$ be the set of utility functions capturing this information and the information that the utility difference between $w$ and $x$ is greater than the difference between $x$ and $y$. Similarly, let $L^{\prime}$ be the set that captures ordinal information plus the fact that the utility difference between $x$ and $y$ is greater than between $y$ and $z$. There are utility functions compatible with the union of this information, which will be members of the intersection of $L$ and $L^{\prime}$, and there are utility functions compatible with one of the pieces of 
extra information but not the other (so $L$ is not a subset of $L^{\prime}$ and vice versa).

Furthermore, if more information is added then the set of feasible utility functions is restricted and so $L$ can be a subset of another set $L^{\prime}$.

The question arises as to how to aggregate the available information in this environment. If comparability of differences is meaningful then it may be desirable to choose between states based upon the utilitarian sum of utilities. The situation is a variant of the one we investigated in section 7.1. If a social ranking is made only when all utility functions in the set L imply the same ranking in terms of the sum of utilities, the approach adopted by Sen (1970b), then the social ranking will generate an incomplete ordering - a quasi-ordering.

The alternative approach is to use available (partially comparable) information to estimate the expected sum of utility difference between two states and use this to generate a complete social ordering. Again, this will involve a degree of conjecture. This suggests that partial comparability should be captured by a probability distribution over utility functions capturing degrees of belief. This could be a degree of belief over sets of utility functions, each set being a partition of the set of functions that occurs under comparability of differences. In this framework, one extreme is to base aggregate judgements on the expected sum of utilities and create a complete ordering. The other extreme is to be risk averse and rank states only when it is certain that the sum of utilities is higher in one state than another. The intermediate case, with merit, is to rank states only when the probability distribution over the sum of differences is either predominantly in the positive range or predominantly in the negative range. Further development of this sort of approach would be useful.

\section{Informational Bases and Welfarism}

The characterization results under alternative informational bases that were discussed in Section 5, all embody the Pareto criterion and lead to rules that can be termed, to generalize Sen's (1979) definition, strict-ranking welfarism - the welfare of each state is evaluated using a function of utilities and the social ranking corresponds to the welfare ranking whenever welfare is not the same in two states. Thus, non-utility information will usually be excluded from the social ranking. 
Whilst it is generally recognised that the Pareto criterion conflicts with the use of nonutility information (Sen (1970a)), it is not well recognised that the ability to make judgements incorporating both utility and non-utility information requires not only an abandonment of the Pareto criterion but also places demands on the richness of utility information that must be available either directly or indirectly through some estimation exercise.

What happens if condition $(P)$ is not imposed? In the Arrow set-up, Wilson (1972) has shown that conditions $(U),(I)$ and $(N D)$ imply either that a pair of states is ranked independently of preferences or there is a 'reverse dictator', where the social ranking is the reverse of the of the ranking of the individual who is the reverse dictator. In the first case, utility information is ignored, in the second non-utility information is ignored and individual utilities are viewed as a "bad".

To rule out utilities as a "bad", a monotonicity condition can be imposed:

Monotonicity $\left(M^{*}\right)$. For all $u, u^{\prime}$ and $x, y \in X$ such that

$$
\begin{aligned}
& u^{\prime}(z, i)=u(z, i) \text { for all } i \in N, \text { for all } z \neq x, y \\
& u^{\prime}(x, i)>u(x, i) \text { for all } i \in N, \\
& u^{\prime}(y, i)<u(y, i) \text { for all } i \in N,
\end{aligned}
$$

$x R y \Rightarrow x P^{\prime} y$ where $R=f(<u>)$ and $R^{\prime}=f\left(<u^{\prime}>\right)$.

Monotonicity allows for a general bias for some state $y$ over state $x$ but, if utility information is such as to create an aggregate judgement for $x$ over $y$, then this is reinforced when utilities move further in favour of $x$ and against $y$. In the Arrow set-up, conditions $(U),(I),\left(M^{*}\right)$ and $(N D)$ imply that utility information must be ignored in the social ranking. Thus, strict-ranking welfarism can be avoided by relaxing the condition $(P)$ but at the cost of adopting welfare indifference with no concern for individual utilities!

This striking result is a consequence of the utility informational base in the Arrow problem. To see this, assume that we move to the other extreme - total comparability - where 
utilities can be evaluated against an independent yardstick. Assume that social states are given some weight $w(x), x \in X$, independently of utilities, and that the social judgement takes the form of ranking $x$ over $y$ if the sum of utilities in state $\mathrm{x}$ plus $\mathrm{w}(\mathrm{x})$ is greater than the sum of utilities in state $y$ plus $w(y)$. This rule satisfies $\left(U^{*}\right),\left(I^{*}\right),\left(M^{*}\right)$ and $\left(N D^{*}\right)$ and allows a trade-off between utility and non-utility information, e.g. the function $w($.$) may be$ inversely related to the degree of coercion that exists in any social state. ${ }^{14}$

Now assume that the appropriate informational base is that of full comparability with comparability of utility levels and differences. To begin, let us consider what is possible in a one-person society; full comparability is equivalent to a cardinal utility function for the individual. With condition $\left(I^{*}\right)$, the only relevant utility information to determine the ranking over a pair of states $\{x, y\}$ is the individual's ordinal ranking of the pair. The ranking of $x$ and $y$ will either follow this individual ranking or ignore it; whatever, there can be no trade-off between utility and non-utility information.

In a many-person society, non-utility information can interact with utility information only through the trade-off between individual utilities. The full proof of this result will not be presented here. ${ }^{15}$ Instead, consider a situation where the set of social states $X$ can be partitioned into subsets $X_{1}, X_{2}, \ldots$ such that if $x, y \in X_{i}$ then these states have the same nonutility characteristics and, to compare $x$ and $y$, only utility information is used to obtain a ranking. Then the aggregation rule to be applied over $X_{i}$ will incorporate the Pareto criterion and, as discussed earlier, it must be welfarist. Also, whenever there is a subset of states such that everybody has the same utility level in those states then a Wilson-type result applies over this subset. Consider $x, y \in X_{1}$ and $x^{\prime}, y^{\prime} \in X_{2}$ such that everybody has the same utility level in state $y$ and everybody has the same level in state $y^{\prime}$. The ranking between $x$ and $y$, and between $x^{\prime}$ and $y^{\prime}$ will be welfarist, the ranking between $y$ and $y^{\prime}$ will be either welfarist, insuring the ranking between $x$ and $x^{\prime}$ is essentially welfarist, or the ranking between $y$ and $y^{\prime}$ will be fixed independently of utilities - in which case all elements of $X_{1}$ are always ranked above the elements of $X_{2}$ or vice versa.

\footnotetext{
${ }_{15}^{14}$ But note that there may be restrictions on the measurability of non-utility information.

15 The proof builds upon the characterization of welfarist objectives under full comparability (Roberts (1980a).
} 
To give an example of what is achievable, let us focus on the case where weighted utilitarianism is to be applied between states with the same non-utility characteristics. Let the weight on individual $i$ be $\gamma_{i}^{j}$ over $X_{j}$. The ranking of states will follow the function

$$
W_{j}=\sum_{i=l, n} \gamma_{i}^{j} u(x, i)
$$

Now, between two states in different partitions, the ranking will either be dictated by nonutility characteristics or, if utility information is to be relevant, it is necessary that if $u(x, i)=\mathrm{u}$ for all $i$ and $u(w, i)=\mathrm{u}^{\prime}$ for all $i$ and $\mathrm{u}>\mathrm{u}^{\prime}$ then $x$ will be preferred. This is ensured if the weights satisfy $\sum_{\mathrm{i}} \gamma_{\mathrm{i}}^{\mathrm{j}}=1$. This serves to characterize the aggregation mechanism - states are to be judged by the weighted average utility level in the state and non-utility information can determine the weights to be used. Thus, there is no possible trade-off between utility and non-utility information other than that the trade-off between two individuals' utilities can be determined by non-utility information.

If levels of utility are not interpersonal comparable then this limited possibility of combining utility and non-utility information is not feasible. To see this, note first that comparability of differences only implies that, within each partition, the rule must be weighted utilitarian. ${ }^{16}$ If utility information is relevant to rank states in partition $j$ with states in partition $k$ then the ranking will be based upon weighted utilitaranism with $\sum_{i} \gamma_{i}{ }^{j}=1$. Assume that $\gamma_{1}{ }^{\mathrm{j}}>\gamma_{1}{ }^{\mathrm{k}}$ and $\gamma_{2}{ }^{\mathrm{j}}>\gamma_{2}{ }^{\mathrm{k}}$. By adding a large enough constant to 1 's utility, states in partition $j$ will be preferred; by adding a large enough constant to 2's utility, states in partition $k$ will be preferred. However, if there is no comparability of levels then the social choice should be invariant with respect to the addition of constants. Thus the weights must be equal across a group of partitions where rankings can depend upon welfare information: i.e. $\gamma_{i}^{j}=\gamma_{i}{ }^{k}{ }^{17}$

A variable weighted utilitarian rule, with weights relating to non-utility information, requires comparability of differences and levels to be implemented. With only comparability of differences, the weights must be unvarying and non-utility information cannot be combined with utility information to create a social ranking.

\footnotetext{
${ }^{16}$ Recall the characterization result of d'Aspremont and Gevers (1977).

${ }^{17} \mathrm{~A}$ formal proof is close to the proof of Theorem 9 of Roberts (1980a).
} 
On the other hand, if there is comparability of utility levels but no comparability of differences then non-utility information can help determine the utility trade-off. For instance, the welfare of each state can be associated with the utility at some position in the utility hierarchy but the position chosen can be state dependent. In coercive states of the world, welfare could be associated with the utility of the worst off individual; in states where there is considerable freedom, welfare could be associated with the utility of the individual with median utility. Such a rule - a state dependent positional dictatorship incorporates a preference for freedom, but only when there is utility inequality, and satisfies $\left(U^{*}\right),\left(I^{*}\right),\left(M^{*}\right)$ and $\left(N D^{*}\right)$ under the informational base of level comparability.

\section{Concluding Remarks}

Following Arrow's pioneering formulation of a structure to analyze social choice, Sen has made fundamental contributions in enriching the structure both with the introduction of social welfare functionals, and with insightful analyses of the information on which social welfare functionals can bear.

Arrow's impossibility theorem is commonly viewed as a result of trying to do too much with too little information and, indeed, with interpersonally comparable information, aggregation mechanisms that satisfy the spirit of his axioms can be formulated. This essay has examined Sen's informational enrichment approach, this being one part of his overall contribution to the analysis of social choice. It has been shown that whilst the use of interpersonal comparisons permits the implementation of whole classes of aggregation mechanisms, there are still severe restrictions on aspects of these mechanisms. Even with comparability of levels and of differences, aggregation mechanisms are restrictive, both in terms of the way that the distribution of utilities can be assessed, and in terms of the way that utility and non-utility information can interact when welfarist conditions like the Pareto criterion are relaxed. To overcome these problems, rich informational structures are required and they come from using all available information to estimate interpersonal comparisons with the required degree of richness. 


\section{References}

Arrow, K. J. (1951). Social Choice and Individual Values (New York: Wiley), 2nd edition 1963.

d'Aspremont, C. and L. Gevers (1977). 'Equity and the Informational Base of Collective Choice', Review of Economic Studies, 46, 199-210.

Basu, K. (1983). 'Cardinal Utility, Utilitarianism, and a Class of Invariance Axiomsis in Welfare Analysis,' Journal of Mathematical Economics, 12, 193-206.

Bossert, W. (1991). 'On Intra-and Interpersonal Utility Comparisons,' Social Choice and Welfare, 8, 207-219.

Deschamps, R. and L. Gevers (1978). 'Leximin and Utilitarian Rules: A Joint Characterization', Journal of Economic Theory, 17, 143-63.

Fleurbaey, M. (2003). 'On the Informational Basis of Social Choice', Social Choice and Welfare, 21, 347-384.

Gevers, L. (1979). 'On Interpersonal Comparability and Social Welfare Orderings', Econometrica, 47, 75-90.

Hammond, P. J. (1975). 'Equity, Arrow's Conditions and Rawls' Difference Principle', Econometrica, 44, 793-804.

Parks, R. P. (1976). 'An Impossibility Theorem for Fixed Preferences: A Dictatorial Bergson-Samuelson Welfare Function', Review of Economic Studies, 43, 447-50.

Pazner, E. (1979). ' Equity, Nonfeasible Alternatives and Social Choice: a Reconsideration of the Concept of Social Welfare', in Aggregation and Revelation of Preferences (J.-J. Laffont (ed.)), Amsterdam:North-Holland. 
Robbins, L. (1935). An Essay in the Nature and Significance of Economic Science, 2nd ed., London: Macmillan.

Roberts, K. W. S. (1980). 'Possibility Theorems with Interpersonally Comparable Welfare Levels', Review of Economic Studies, 47, 409-20.

Roberts, K. W. S. (1980a). 'Interpersonal Comparability and Social Choice Theory', Review of Economic Studies, 47, 421-39.

Roberts, K. W. S. (1980b). 'Social Choice Theory: The Single and Multi-Profile Approaches', Review of Economics Studies, 47, 441-450.

Roberts, K. W. S. (1997). 'Comment on Suzumura on Interpersonal Comparisons', in Social Choice Re-Examined, Vol.2 (Arrow, Sen and Suzumura, eds.) London: Macmillan.

Sen, A. K. (1966). 'A Possibility Theorem on Majority Decisions', Econometrica, 34, 481-9.

Sen, A. K. (1970). Collective Choice and Social Welfare (San Francisco: Holden-Day)

Sen, A. K. (1970a). 'The Impossibility of a Paretian Liberal', Journal of Political Economy, $78,152-7$.

Sen, A.K. (1970b). 'Interpersonal Aggregation and Partial Comparability', Econometrica, 38, 393-409.

Sen, A. K. (1977). 'On Weights and Measures: Informational Constraints in Social Welfare Analysis', Econometrica, 45, 1539-72.

Sen, A. K. (1979). 'Personal Utilities and Public Judgements or What's Wrong with Welfare Economics', Economic Journal, 89, 537-58.

Sen, A. K. (1999) 'The Possibility of Social Choice', American Economic Review, 89, 349-78. 
Sen, A. K. and P. K. Pattanaik (1969). 'Necessary and Sufficient Conditions for Rational Choice under Majority Decision', Journal of Economic Theory, 1, 178-202.

Strasnick, S. (1976). 'Social Choice Theory and the Derivation of Rawls' Difference Principle', Journal of Philosophy, 73, 85-99.

Wilson, R. B. (1972). 'Social Choice without the Pareto Principle', Journal of Economic Theory, 5, 478-486. 